BULLETIN (New Series) OF THE

AMERICAN MATHEMATICAL SOCIETY

Volume 42, Number 1, Pages 57-78

S 0273-0979(04)01045-6

Article electronically published on October 29, 2004

\title{
RECENT PROGRESS ON THE POINCARÉ CONJECTURE AND THE CLASSIFICATION OF 3-MANIFOLDS
}

\author{
JOHN W. MORGAN
}

\section{INTRODUCTION}

Motivated by what was well-known at the time about surfaces, and after a long topological study of 3-manifolds, at the very end of his 1904 paper Poincaré 18 states that there remains one question to treat, namely (reformulated in modern language):

If $M$ is a closed 3-manifold with trivial fundamental group, then is $M$ diffeomorphid ${ }^{1}$ to $S^{3}$ ?

The Poincaré Conjecture is that the answer to this question is "Yes." Developing tools to attack this problem formed the basis for much of the work in 3 -dimensional topology over the last century, including for example, the proof of Dehn's lemma and the loop theorem and the study of surgery on knots and links.

In the 1980's Thurston developed another approach to 3-manifolds, see 24 and [4]. He considered 3-manifolds with riemannian metrics of constant negative curvature -1 . These manifolds, which are locally isometric to hyperbolic 3-space, are called hyperbolic manifolds. There are fairly obvious obstructions showing that not every 3 -manifold can admit such a metric. Thurston formulated a general conjecture that roughly says that the obvious obstructions are the only ones; should they vanish for a particular 3-manifold then that manifold admits such a metric. His proof of various important special cases of this conjecture led him to formulate a more general conjecture about the existence of locally homogeneous metrics, hyperbolic or otherwise, for all manifolds; this is called Thurston's Geometrization Conjecture for 3-manifolds. The statement of this conjecture is somewhat complicated, so it is deferred until Section 2. An important point is that Thurston's Geometrization Conjecture includes the Poincaré Conjecture as a very special case. In addition, Thurston's conjecture has two advantages over the Poincaré Conjecture:

- It applies to all closed oriented 3-manifolds.

- It posits a close relationship between topology and geometry in dimension three.

Received by the editors June 11, 2004, and, in revised form, September 1, 2004.

2000 Mathematics Subject Classification. Primary 57M50, 57M27, 58J35.

Written version of a talk presented on January 9, 2004, in the "Current Events in Mathematics" session at the AMS national meeting in Phoenix, AZ.

${ }^{1}$ Every topological 3-manifold has a differential structure unique up to diffeomorphism close to the identity. Hence, for 3-manifolds classifications up to homeomorphism and up to diffeomorphism are equivalent. 
In fact, it is my view that before Thurston's work on hyperbolic 3-manifolds and his formulation of his general Geometrization Conjecture there was no consensus among the experts as to whether the Poincaré Conjecture was true or false. After Thurston's work, notwithstanding the fact that it has no direct bearing on the Poincaré Conjecture, a consensus developed that the Poincaré Conjecture (and the Geometrization Conjecture) were true. Paradoxically, subsuming the Poincaré Conjecture into a broader conjecture and then giving evidence, independent from the Poincaré Conjecture, for the broader conjecture led to a firmer belief in the Poincaré Conjecture. For more details on the history of the Poincaré Conjecture, the development of 3-manifold topology, and Thurston's Geometrization Conjecture, see Milnor's survey article 13.

The aspect of Thurston's Geometrization Conjecture that is most relevant for us is that the conjectural existence of especially nice metrics on 3-manifolds suggests a more analytic approach to the problem of classifying 3-manifolds. Hamilton [5] formalized one such approach by introducing the Ricci flow on the space of riemannian metric 3 (on a manifold of any dimension). He then conjectured that starting with any riemannian metric on a compact 3 -manifold, the Ricci flow should produce a one-parameter family of metrics converging to the nice metric as postulated by Thurston's Geometrization Conjecture. But there are many technical issues with this program: One knows that in general the Ricci flow will develop singularities in finite time, and thus a method for analyzing these singularities and continuing the flow past them must be found. Furthermore, even if the flow continues for all time, there remain complicated issues about the nature of the limiting object at time $t=+\infty$.

Hamilton [5, 6, 7, 8, 9] made significant progress on the program he initiated by establishing many crucial analytic estimates for understanding the evolving metric and its curvatures. He also showed by applying geometric limit arguments à la Cheegar-Gromov [2] that one could, in special cases, use the Ricci flow to establish Thurston's Geometrization Conjecture. More precisely, he was able to show with certain hypotheses, including that the Ricci flow does not develop singularities in finite time, that the family of metrics as $t \mapsto \infty$ can be rescaled so as to converge to a complete hyperbolic metric; hence the manifold in question has a hyperbolic metric. More recently, building on this work, Perelman, in a series of three preprints 15, 16, 17, has claimed to surmount all of the various technical, geometric and analytic difficulties of Hamilton's program. The basis for Perelman's results begins with a detailed analysis of the regions in a Ricci flow where the curvature is large. He gives a nice classification of such regions, sufficient to allow him to analyze the finite time singularities occurring in a Ricci flow and to give a rough classification of these. Using this he shows how to extend the flow in a natural way past the singularities, constructing what he calls the Ricci flow with surgery. (Hamilton had previously defined an analogous surgery process in the context of 4-manifolds.) Perelman has extended many of Hamilton's estimates for the Ricci flow to the Ricci flow with surgery. By using these techniques, Perelman is able to continue the flow and the surgery process for all positive time. Lastly, he extended Hamilton's analysis of the limits as $t \mapsto \infty$ to the Ricci flow with surgery. In this way he claims to have established Thurston's Geometrization Conjecture and hence the Poincaré Conjecture. Perelman's arguments are quite intricate and involve many strikingly

\footnotetext{
${ }^{2}$ The Ricci flow on metrics is defined in Section 3.
} 
original ideas. The mathematical community is still trying to digest his argument and ascertain whether it is indeed a complete and correct argument. As of August, 2004, the arguments have held up under this scrutiny. While the analysis of his arguments is not yet complete, it is clear that Perelman has made tremendous advances in understanding the nature of Ricci flow for 3-manifolds.

\section{LOCALLY HOMOGENEOUS MANIFOLDS}

Let us set the stage for Hamilton's and Perelman's work. More detailed references for locally homogeneous manifolds, especially in dimension three, are 24, 19] and [4].

1.1. Homogeneous Geometries and Geometric Structures. A homogeneous riemannian manifold $(M, g)$ is one whose group of isometries acts transitively on the manifold. This means that the manifold looks the same metrically at any point. Examples of homogeneous manifolds are the round sphere $S^{n}$, Euclidean space $\mathbb{R}^{n}$, and hyperbolic space $\mathbb{H}^{n}$. The latter is the hypersurface in $\mathbb{R}^{n+1}$ given by $\{Q(x)=$ $-1\}$ where $Q\left(x_{0}, \ldots, x_{n}\right)=-x_{0}^{2}+x_{1}^{2}+\cdots+x_{n}^{2}$. The riemannian metric is the one induced by restricting $Q$ to the tangent planes to the hypersurface. The subgroup of index two in the orthogonal group of $Q$ (consisting of all transformations that leave invariant the upper sheet of the hyperbola) acts transitively as the full group of isometries of $\mathbb{H}^{n}$. All the sectional curvatures of $\mathbb{H}^{n}$ are equal to -1 .

We say that a riemannian manifold is modeled on a given homogeneous manifold $(M, g)$ if every point of the manifold has a neighborhood isometric to an open set in $(M, g)$. Such manifolds are called locally homogeneous manifolds provided that they are complete. The universal covering of a locally homogeneous manifold (with its induced riemannian metric) is a homogeneous manifold that it is modeled on. We are primarily concerned with locally homogeneous manifolds of finite volume.

In dimension 2 there are four simply connected homogeneous manifolds up to isometry: $S^{2}, \mathbb{R}^{2}, \mathbb{H}^{2}$, and $G$, with a left-invariant metric, where $G$ is the group $\mathbb{R} \ltimes \mathbb{R}^{*}$ with the natural action of $\mathbb{R}^{*}$ on $\mathbb{R}$. It turns out that there are no finite volume locally homogeneous manifolds modeled on the fourth example, and hence we are concerned with only the first three models. The finite volume locally homogeneous manifolds of the first type are the sphere and the projective plane, of the second type are the torus and the Klein bottle, and of the third type are complete hyperbolic surfaces of finite volume which can be either compact or non-compact. Of course, for the latter two types, there are continuous families of non-isometric locally homogeneous metrics on these manifolds.

This gives us the following well-known and classical theorem.

Theorem 1.1.1 (Uniformization in dimension 2). Let $X$ be a compact surface. Then $X$ admits a locally homogeneous metric locally isometric to one of the constant curvature models above. The model will be positively curved if $\chi(X)>0$, flat if $\chi(X)=0$, and negatively curved or hyperbolic if $\chi(X)<0$.

1.2. Dimension 3. In dimension three, every finite volume, locally homogeneous manifold is modeled on one of the eight homogeneous manifolds listed below (see [24, pp. 179-190], [19] and [4] for more details). (As in dimension two, there are other homogeneous three-dimensional manifolds, but there are no finite volume locally homogeneous manifolds modeled on them.) First, we have the constant (sectional) curvature examples: 
- $S^{3}$ of constant curvature +1 .

- $\mathbb{R}^{3}$, which is flat.

- $\mathbb{H}^{3}$ of constant curvature -1 .

Any locally homogeneous manifold modeled on the first is a riemannian manifold of constant positive sectional curvature. These are of the form $S^{3} / \Gamma$ where $\Gamma$ is a finite subgroup of $S O(4)$ acting freely on $S^{3}$ and include $S^{3}, \mathbb{R} P^{3}$, lens spaces, as well as the quotients by the symmetry groups of the exceptional regular solids. These manifolds are called spherical space-forms. Notice that all spherical spaceforms are compact.

The prototypical compact flat 3-manifolds are flat tori. These are the quotients of $\mathbb{R}^{3}$ by a full (i.e., rank 3 ) lattice of translations. Any locally homogeneous manifold modeled on $\mathbb{R}^{3}$ has a flat metric. If it is of finite volume, then it is finitely covered by a flat $T^{3}$ and hence is automatically compact.

A locally homogeneous manifold modeled on $\mathbb{H}^{3}$ is a complete, hyperbolic manifold, i.e., a riemannian manifold with all sectional curvatures equal to -1 that is complete. Up to diffeomorphism, there are infinitely many such manifolds of finite volume both compact and non-compact. By Mostow rigidity, a 3-manifold admits at most one (up to isometry) complete, finite volume hyperbolic metric. If the manifold in question is not compact, then each component of a neighborhood of infinity is diffeomorphic to $T^{2} \times(1, \infty)$. Geometrically the torus slices become small exponentially fast as $t$ increases. In particular, any finite volume hyperbolic 3-manifold is diffeomorphic to the interior of a compact 3-manifold with the property that every boundary component is a two-torus whose fundamental group injects into that of the manifold.

Next we have the homogeneous 3-manifolds with product metrics:

- $S^{2} \times \mathbb{R}$.

- $\mathbb{H}^{2} \times \mathbb{R}$.

Finite volume locally homogeneous manifolds modeled on $S^{2} \times \mathbb{R}$ are automatically compact and either are isometric to $S^{2} \times S^{1}$ or $\mathbb{R} P^{3} \# \mathbb{R} P^{3}$. Finite volume locally homogeneous manifolds modeled on $\mathbb{H}^{2} \times \mathbb{R}$ either are of the form $\Sigma \times S^{1}$, where $\Sigma$ is a finite area hyperbolic surface, or are finitely covered by such manifolds. In the later case, the manifold is Seifert fibered over a hyperbolic two-dimensional orbifold of finite area. (See [10] or [20] for more details on Seifert fibered 3-manifolds, and for the definition and the basic facts about orbifolds see [4.) The examples Seifert fibered over a hyperbolic base can be non-compact, but then they are diffeomorphic to interiors of compact manifolds with every boundary component being a torus. Again, the fundamental group of each boundary component injects into that of the manifold.

Lastly, we have the homogeneous manifolds $(M, g)$ where $M$ is a simply connected Lie group and $g$ is a left-invariant metric. The group acts isometrically on itself by left multiplication and hence is embedded in the group of isometries of $M$. Three-dimensional examples of this type admitting locally homogeneous examples of finite volume are:

- The unipotent group (Heisenberg group) of strictly upper triangular threeby-three matrices $N^{3}$. Locally homogeneous manifolds modeled on this group are called nil-manifolds. Any finite volume nil-manifold is finitely covered by a manifold fibered by circles over the two-torus and, in particular, is compact. 
- The solvable group is written as a semi-direct product $\mathbb{R}^{2} \ltimes \mathbb{R}^{*}$ where the action of $t \in \mathbb{R}^{*}$ on $\mathbb{R}^{2}$ is diagonal and given by the matrix

$$
\left(\begin{array}{cc}
t & 0 \\
0 & t^{-1}
\end{array}\right)
$$

Locally homogeneous manifolds modeled on this group are called solvmanifolds. Solv-manifolds of finite volume are fibered by two-tori over the circle or are finitely covered by such manifolds. Again, all these examples are compact.

- $G=\widetilde{P S L}_{2}(\mathbb{R})$, the universal covering group of $P S L_{2}(\mathbb{R})$. This manifold can also be viewed as the universal covering of the unit tangent bundle to $\mathbb{H}^{2}$ with its induced metric. Finite volume locally homogeneous manifolds modeled on this example are circle bundles over hyperbolic surfaces or more generally Seifert fiber bundles over hyperbolic orbifolds. These examples can be non-compact, but if so they are diffeomorphic to the interior of a compact manifold all of whose boundary components are tori. The fundamental groups of these tori inject into the fundamental group of the manifold.

Summarizing the central features common to all these examples, we have:

Corollary 1.2.1. Suppose that $X^{3}$ is connected and orientable and admits a locally homogeneous riemannian metric of finite volume. Then, $X$ is diffeomorphic to the interior of a compact 3-manifold with boundary, all of whose boundary components are tori. Furthermore, each of these tori has fundamental group which injects into the fundamental group of $X$. If $X$ is non-compact, then it is modeled on $\mathbb{H}^{3}, \mathbb{H}^{2} \times \mathbb{R}$ or $\widetilde{P S L}_{2}(\mathbb{R})$, and hence, $X$ either is a hyperbolic 3-manifold or is Seifert-fibered with hyperbolic two-dimensional orbifold base.

The converse for Seifert fibered 3-manifolds is also important in our story.

Theorem 1.2.2. Let $X$ be a Seifert fibered 3-manifold (with boundary). The base of the Seifert fibration is a compact 2-dimensional orbifold, possibly with boundary. If the orbifold Euler characteristic of the base orbifold is less than zero, then the interior of $X$ admits a finite volume locally homogeneous metric modeled on $\mathbb{H}^{2} \times \mathbb{R}$ or $\widetilde{P S L}_{2}(\mathbb{R})$. If the Euler characteristic of the base is zero, then either $X$ admits a locally homogeneous metric modeled on $\mathbb{R}^{3}$ or $N^{3}$ or $X$ is diffeomorphic to $T^{2} \times I$ or the twisted I-bundle over the Klein bottle. If the Euler characteristic of the base is positive, then either $X$ has a locally homogeneous metric modeled on $S^{2} \times \mathbb{R}$ or $S^{3}$ or $X$ is a solid torus.

Corollary 1.2.3. Let $X$ be the interior of a 3-manifold $\bar{X}$ (possibly with boundary) that Seifert fibers over a compact 2-dimensional orbifold. Then $X$ admits a locally homogeneous metric of finite volume unless $\bar{X}$ is diffeomorphic to $T^{2} \times I$, a twisted I-bundle over the Klein bottle, or the solid torus.

\section{TOPOLOGY OF 3-MANIFOLDS}

There are several reasons that, unlike the case in dimension two, not every compact 3-manifold can admit a homogeneous metric of finite volume. The most obvious reason has to do with the fact that 3-manifolds are not necessarily prime, whereas, with one exception, all locally homogeneous 3-manifolds are prime. 

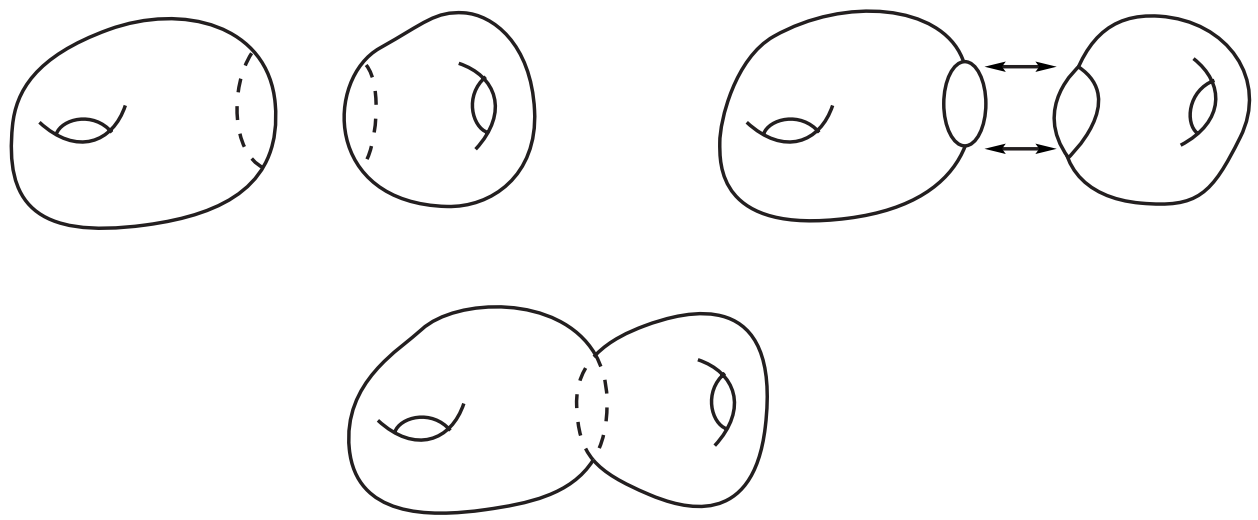

Figure 1. Connected sum.

2.1. The Prime Decomposition. Let us recall the prime decomposition of 3manifolds.

Definition 2.1.1. Given connected, oriented smooth $n$-manifolds $X$ and $Y$ we define their connected sum as follows. Remove from each of $X$ and $Y$ the interior of a compact $n$-ball that is contained in the interior of the manifold. Denote by $X_{0}$ and $Y_{0}$ the resulting manifolds with distinguished boundary components which are $(n-1)$-spheres. Then glue these spheres together in such a way that the orientations on $X_{0}$ and $Y_{0}$ match along the common $(n-1)$-sphere. The result admits a smooth structure compatible with those of $X_{0}$ and $Y_{0}$ which, up to diffeomorphism, is independent of the choice of embeddings of the $n$-balls and the gluing isomorphism. It is called the connected sum of $X$ and $Y$. (See FIG. 1.) Notice that the connected sum of $X$ and an $n$-sphere is diffeomorphic to $X$. A 3-manifold $X$ is said to be prime if it is not diffeomorphic to $S^{3}$ and if every $S^{2} \subset X$ that separates $X$ into two pieces has the property that one of the two pieces is diffeomorphic to a three-ball. Equivalently, anytime we write $X$ as a connected sum of two manifolds exactly one of them must be the three-sphere.

One of the first theorems in the topology of 3-manifolds is due to Kneser in 1929 11]:

Theorem 2.1.2. Every closed, oriented 3-manifold admits a decomposition as a connected sum of oriented prime 3-manifolds, called its prime factors. This decomposition is unique up to the order of the prime factors (and orientation-preserving diffeomorphism of the factors). There are a countably infinite number of prime 3-manifolds up to diffeomorphism.

An essential $S^{2} \subset X$ is a two-sphere that does not bound a three-ball in $X$. If this sphere separates, then cutting $X$ along it and filling in the holes with threeballs implements a connected sum decomposition of $X$. If on the other hand a $S^{2} \subset X$ does not separate, then this procedure has the effect of removing from $X$ a prime factor diffeomorphic to $S^{2} \times S^{1}$.

Because of the prime decomposition, all questions about the topology of closed, oriented 3-manifolds can be reduced to questions about prime 3-manifolds. For example: 
Corollary 2.1.3. $\Sigma^{3}$ is a homotopy three-sphere if and only if all its prime factors are homotopy three-spheres. Thus, there is a counterexample to the Poincaré Conjecture if and only if there is a counterexample that is a prime 3-manifold.

By the sphere theorem, if $\pi_{2}(M) \neq 0$, then $X$ contains an essential $S^{2}$. The converse is equivalent to the Poincaré Conjecture.

2.2. Thurston's Geometrization Conjecture. Excluding locally homogeneous manifolds modeled on $S^{2} \times \mathbb{R}$, all locally homogeneous 3-manifolds have universal coverings which are diffeomorphic either to $\mathbb{R}^{3}$ or $S^{3}$. It is easy to see that if every embedded 2 -sphere in the universal covering of a 3 -manifold bounds a threeball, then the same is then true in the original manifold. Thus, the only locally homogeneous 3-manifolds with essential $S^{2}$ 's are those whose universal cover is $S^{2} \times \mathbb{R}$. These are $S^{2} \times S^{1}$ and $\mathbb{R} P^{3} \# \mathbb{R} P^{3}$, the latter being the only example of a non-prime manifold admitting a locally homogeneous metric. Hence, if we are interested in imposing locally homogeneous metrics, then we must restrict our attention to prime 3 -manifolds.

Thurston's Geometrization Conjecture is about prime manifolds; see [4].

Conjecture 2.2.1. (Thurston's Geometrization Conjecture) Let $M$ be a closed, orientable, prime 3-manifold. Then there is an embedding of a disjoint union of 2-tori and Klein bottles $\coprod_{i} T_{i}^{2} \subset M$ such that every component of the complement admits a locally homogeneous riemannian metric of finite volume.

The locally homogeneous metrics on the various components of this cutting process can be modeled on different homogeneous manifolds.

Since we are working with orientable manifolds, the boundary of a neighborhood of a Klein bottle is a two-torus, so that the resulting geometric pieces are interiors of compact manifolds all of whose boundary components are tori. Notice that there can in general be more than one such family of tori and Klein bottles up to isotopy for which the conclusion of the Geometrization Conjecture holds. For example, let $\Sigma_{2}$ be a surface of genus 2 . Then $\Sigma_{2} \times S^{1}$ has a locally homogeneous metric. Let $T^{2} \subset \Sigma_{2} \times S^{1}$ be the torus lying over a loop in $\Sigma_{2}$ separating it into two once-punctured tori. Then each of the two components of $\Sigma_{2} \times S^{1} \backslash T$ also has a finite volume locally homogeneous metric. To obtain uniqueness one takes a family in $X$ satisfying the conclusion with a minimal number of components. With this condition, the family is unique up to isotopy.

A closed surface of genus at least one in a 3-manifold is said to be incompressible if its fundamental group injects into the fundamental group of the 3-manifold. Notice by Corollary 1.2.1 each torus boundary component has fundamental group injecting into each of the 3-manifolds that it bounds. It follows from Van Kampen's theorem that the fundamental group of each torus injects into $\pi_{1}(M)$; that is to say these tori are incompressible. By similar reasoning the Klein bottles also are incompressible. Thus, one can formulate Thurston's Geometrization Conjecture for a prime 3-manifold $M$ as saying that there is a decomposition of $M$ along incompressible tori and Klein bottles into pieces whose interiors admit finite volume locally homogeneous metrics. The Geometrization Conjecture for a general compact, orientable 3-manifold is the statement that each of its prime factors satisfies Conjecture 2.2.1 
2.3. Relation to the Poincaré Conjecture and the Spherical Space-Form Question. It is easy to deduce the Poincaré Conjecture from Thurston's Geometrization Conjecture: For suppose that we have a prime homotopy threesphere $\Sigma$ that satisfies the conclusion of the Geometrization Conjecture. Since $\pi_{1}(\Sigma)=\{1\}, \Sigma$ has no incompressible tori and no incompressible Klein bottles, and hence the decomposition of $\Sigma$ referred to in Conjecture 2.2.1 must be trivial. That is to say $\Sigma$ has a locally homogeneous metric. Again, since $\pi_{1}(\Sigma)$ is trivial, the homogeneous model for $\Sigma$ is $\Sigma$ which is compact. The only compact three-dimensional model is $S^{3}$, and consequently, $\Sigma$ is diffeomorphic to $S^{3}$. Notice that this argument (except for the very last step) applies equally well to prime 3manifolds of finite fundamental group. The conclusion is that the Geometrization Conjecture for such manifolds implies that they are three-dimensional spherical space-forms. These were classified by Hopf; see 12 .

\section{HAMILTON'S RiCCi FLOW EQUATION}

Let us now introduce Hamilton's Ricci flow; cf [5]. This is a parabolic evolution equation for a riemannian metric on a manifold. The general form of the equation is

$$
g^{\prime}(t)=F(g(t))
$$

where a solution is a one-parameter family of metrics on the manifold whose time derivative is given by a functional $F(g(t))$. This functional must be of the same tensor type as the metric, i.e., a contravariant symmetric two-tensor, and should (we hope) involve no more than second derivatives of the metric. It must also be natural (i.e., independent of the coordinates used to write the equation locally). This means that $F$ must be a linear combination of the metric $g(t)$ itself and the Ricci curvature $\operatorname{Ric}(g(t))$, described below, of the metric. The equation that Hamilton introduced is:

$$
g^{\prime}(t)=-2 \operatorname{Ric}(g(t))
$$

the Ricci flow equation.

This equation has a scale invariance: Given a flow $g(t)$ of metrics, define a new flow $h(t)$ by

$$
h(t)=\lambda^{2} g\left(\lambda^{-2} t\right) .
$$

It is easy to see that if the original family of metrics satisfies the Ricci flow equation, then so does the new family.

The fixed points for the Ricci flow equation are clearly the Ricci-flat manifolds, those whose Ricci curvature vanishes. Every Ricci flat 3-manifold is in fact flat. More generally, any Einstein manifold (by definition the Ricci curvature tensor of an Einstein manifold is a multiple of the metric tensor) is self-similar under Ricci flow; the family is an expanding family of self-similar manifolds when the multiple is negative and contracting when the multiple is positive. But any Einstein 3manifold is one of constant sectional curvature. Thus, we see a close relationship between the fixed points (up to conformal factor) of the Ricci flow in dimension three and 3-manifolds with homogeneous geometries. This relationship fails to hold in dimensions four and higher: in those dimensions being Einstein does not imply that the manifold is locally homogeneous. 
3.1. Curvature. Recall that, given a riemannian metric, there is a unique symmetric connection on the tangent bundle of the manifold:

$$
\nabla: \text { Vector Fields } \otimes_{\mathbb{R}} \text { Vector Fields } \rightarrow \text { Vector Fields. }
$$

The connection is usually written

$$
\nabla(X \otimes Y)=\nabla_{X}(Y)
$$

Defining

$$
R(X, Y)=\nabla_{X} \circ \nabla_{Y}-\nabla_{Y} \circ \nabla_{X}-\nabla_{[X, Y]}
$$

introduces the curvature operator which is a section of the tensor product of the second exterior power of the cotangent bundle and the bundle of orthogonal endomorphisms of the tangent bundle. One then defines the riemannian curvature tensor

$$
\operatorname{Rm}(X, Y, Z, W)=-\langle R(X, Y) Z, W\rangle .
$$

This is a section of the fourth tensor power of the cotangent bundle. It is skew in the first two variables and skew in the last two variables and symmetric under interchange of variables $(1,2)$ with variables $(3,4)$. Thus, we can view $\mathrm{Rm}$ as a symmetric two-tensor on the second exterior power of the cotangent bundle. The Ricci curvature is the trace on $\mathrm{Rm}$ on the middle two variables. Thus, if $e_{1}, \ldots, e_{n}$ is an orthonormal frame at a point, we have

$$
\operatorname{Ric}\left(e_{i}, e_{j}\right)=\sum_{k=1}^{n} \operatorname{Rm}\left(e_{i}, e_{k}, e_{k}, e_{j}\right)
$$

This is a contravariant symmetric two-tensor on the manifold. In local coordinates, its leading term is a linear expression in the second partial derivatives of the entries of the matrix $g_{i j}$ describing the metric.

3.2. Relation of the Ricci Flow Equation to the Heat Equation. One should view the Ricci flow equation as a generalization in two ways of the heat equation. First, it concerns tensors (symmetric two-tensors) rather than scalar functions. Second, it is a non-linear parabolic equation whose leading term is a 'heat equation' for tensors. Much of the understanding of the Ricci flow equation comes by analogy from results for the heat equation. To indicate the analogy of the Ricci flow equation with non-linear versions of the heat equation, let us write the evolution equation for the scalar curvature $R$ (which by definition is the trace of the Ricci curvature). The equation in dimension three is

$$
R^{\prime}(t)=\Delta R(t)+\frac{2}{3} R^{2}(t)+\left|\operatorname{Ric}^{0}(t)\right|^{2},
$$

where $\mathrm{Ric}^{0}$ is the traceless part of the Ricci curvature. It is clear from this expression that the minus sign in the Ricci flow equation is forced on us - without it we would have non-linear versions of the backwards heat equation which is an illposed equation. The factor of 2 in the Ricci flow is for convenience only; it can be scaled out. 
3.3. Hamilton's First Results. In [5, 7, 6] Hamilton proved:

- Short-time existence and uniqueness. If $g_{0}$ is a smooth metric on a compact manifold, then there is some $\epsilon>0$ depending on $g_{0}$ and a unique solution to the Ricci flow equation defined for $t \in[0, \epsilon)$ with $g(0)=g_{0}$.

- Curvature characterization of singularity formulation. If the solution exists on the time interval $[0, T)$ but does not extend to any strictly larger time interval, then there is a point $x$ in the manifold for which curvature tensor $\operatorname{Rm}(x, t)$ of the metric $g(t)$ is unbounded as $t$ approaches $T$.

3.3.1. The case of non-negative Ricci curvature. In [5] Hamilton also completely analyzed the Ricci flow on 3-manifolds with non-negative Ricci.

Theorem 3.3.1 (Hamilton). Let $X^{3}$ be a compact connected 3-manifold with nonnegative Ricci curvature. Then one of the following happens:

- The Ricci curvature becomes strictly positive for all $t>0$ sufficiently small. In this case, the Ricci flow develops a singularity in finite time. As the singularity develops, the diameter of the manifold goes to zero. Rescaling the evolving family of metrics so that their diameters are one leads to a family of metrics converging smoothly to a metric of constant positive curvature. In particular, the manifold is diffeomorphic to a spherical space-form.

- There is a finite cover of the riemannian manifold which, with the induced metric, is a metric product of a compact surface of positive curvature and $S^{1}$. This remains true for all the riemannian metrics in the Ricci flow. The Ricci flow develops a singularity in finite time, and the manifold in question is diffeomorphic to $S^{2} \times S^{1}$, or $\mathbb{R} P^{3} \# \mathbb{R} P^{3}$.

- The metric is flat and the evolution equation is constant. In this case, of course, the manifold is finitely covered by $T^{3}$.

In particular, all these manifolds satisfy the conclusion of Thurston's Geometrization Conjecture.

3.3.2. Limits as $t \mapsto \infty$. At the other extreme, in 9] Hamilton analyzed, under certain extra assumptions, what happens to the riemannian metric as $t \mapsto \infty$. Hamilton was able to show:

Theorem 3.3.2 (Hamilton). Suppose that the Ricci flow on a compact, connected riemannian 3-manifold $\left(M, g_{0}\right)$ exists for all $t \in[0, \infty)$ and that the normalized curvature $t \cdot \operatorname{Rm}(x, t)$ is bounded as $t \mapsto \infty$. Then one of the following two cases occurs:

(1) The manifold admits a flat metric.

(2) There is a finite set of complete hyperbolic manifolds $H_{i}$ of finite volume, and for each $t>>1$, an embedding $\varphi_{t}: \coprod_{i} H_{i} \rightarrow M$ with the following properties. The pullbacks of the rescaled metrics, $\varphi_{t}^{*}\left(t^{-1} g(t)\right)$, are converging, uniformly on each compact subset of $\coprod_{i} H_{i}$, to a metric of constant negative curvature, i.e., to a scaled version of the hyperbolic metric. The tori in the $H_{i}$ parallel to infinity are mapped to incompressible tori in $M$. The complement of the image of $\varphi_{t}$ has the following structure: All its prime factors are Seifert fibrations with incompressible boundary tori or are solv-manifolds. 
The way that Hamilton shows that the complement of the image of $\varphi_{t}$ is a manifold all of whose prime factors are Seifert fibrations is through the theory of collapsing metrics with bounded curvature. Hamilton showed that as $t \mapsto \infty$ the metrics on the complement of the image of $\varphi_{t}$, rescaled by $t^{-1}$ are collapsing with bounded curvature. Cheegar-Gromov [2] studied manifolds with this property and showed that they have what is called an $F$-structure. This means that locally the manifold has compatible actions by tori of positive dimension. 3-manifolds with $F$-structures are then easily classified by topological techniques. The result is that they are connected sums of Seifert fibered manifolds and solv-manifolds.

Corollary 3.3.3. If the Ricci flow with initial conditions $\left(M, g_{0}\right)$ satisfies the hypothesis of the previous theorem, then $M$ satisfies Thurston's Geometrization Conjecture.

Proof. This is clear under the first possibility stated in the theorem. Suppose that the second holds. The prime decomposition of the complement of the union of the hyperbolic pieces in fact yields a prime decomposition of the manifold $M$. Thus, each of its prime pieces is decomposed along incompressible tori and Klein bottles into pieces that are either hyperbolic or Seifert fibered. Since the tori and Klein bottles are incompressible, it follows from Theorem 1.2.2 that all the Seifert fibered pieces either admit finite volume locally homogeneous metrics or are themselves isomorphic to $T^{2} \times I$ or the twisted $I$-bundle over the Klein bottle. For each piece of the latter two types, we replace its boundary tori by the central torus or Klein bottle. After this modification, we find that all the prime factors of $M$ satisfy the conclusion of Thurston's Geometrization Conjecture.

\section{Perelman's Claims}

In a sequence of three manuscripts [15, 16, 17] posted on the math archive within the last two years, Perelman has given arguments for a sequence of claims about the Ricci flow and its relation to Thurston's Geometrization Conjecture. Here is the general idea.

The finite time singularities occur inside regions of the manifold of two types. The first type comprises components of the manifold whose metrics are shrinking down in a controlled way (for example, components of positive Ricci curvature shrink to a point). Each region of the second type is a long thin tube diffeomorphic to $S^{2}$ times an interval or the union of such a long thin tube with a cap of positive curvature on the end. Perelman's surgery process is to go to the singular time, remove all regions of the first type (which are components of the manifold) and do surgery near the 'large' ends of the long thin tubes to cap them off with standard (or nearly standard) metrics on the disk. The topological effect of these surgeries is to remove some components which are known to satisfy Thurston's Geometrization Conjecture and to do surgery on the other components implementing a connected sum decomposition (which may be trivial since one of the pieces created by the surgery may well be $S^{3}$ ). From this it follows easily that if the manifold after surgery satisfies Thurston's Geometrization Conjecture, then so does the manifold before surgery. One then takes the result of surgery as the initial condition for continuing the Ricci flow. This process can be repeated ad infinitum producing a flow with surgery defined for all positive time with only finitely many surgeries in any finite time interval. Lastly, Perelman claims that Hamilton's result, Theorem[3.3.2, on the 
nature of the limits as $t \mapsto \infty$ has an analogue valid for the Ricci flow with surgery, even without the assumption on the normalized curvature. Since the manifolds associated to sufficiently large time satisfy Thurston's Geometrization Conjecture, working backwards through the surgeries we see that the initial manifold does also. It is in this way that Perelman claims to establish Thurston's Geometrization Conjecture and consequently the Poincaré Conjecture.

If one is only interested in the Poincaré Conjecture, or more generally manifolds with finite fundamental group, then according to Perelman things simplify even more, for the Ricci flow with surgery becomes extinct after a finite time and there is no need for the analogue of Theorem 3.3 .2

For simplicity of the exposition only, we assume henceforth that our 3manifolds admit no embedded projective planes. For the topological results this is not a serious restriction since a neighborhood of an embedded $\mathbb{R} P^{2}$ in an orientable manifold is $\mathbb{R} P^{3} \backslash D^{3}$ and hence there is a connected sum decomposition of the manifold splitting off an $\mathbb{R} P^{3}$. After splitting off finitely many such $\mathbb{R} P^{3}$ factors, the result contains no embedded $\mathbb{R} P^{2}$.

4.1. The Surgery Parameters. In doing surgery there are three parameters that are fixed. The first is the coarse control parameter $\epsilon>0$, sufficiently small, a universal constant fixed once and for all. The other two parameters are non-increasing functions of $t$ limiting to zero as $t \mapsto \infty$. They are $\delta(t)>0$, the fine control parameter, and $h(t)$, the scale parameter. We also fix an auxiliary parameter $\rho(t)=\epsilon \cdot \delta(t)$. Whenever we do surgery at time $t$ we use $\epsilon$ and the values of these parameters at time $t$ in order to do surgery. Perelman's claim is that if these constants and functions are chosen correctly, the results below are valid. They are now taken as fixed.

4.2. Regions of High Curvature in the Flow. In order to do surgery we need to understand regions of the flow where the scalar curvature is large. Of course, since it is possible to rescale the metric and time in any flow, we must normalize in some fashion to have an invariant notion of large curvature. Thus, we arrange that our flow has normalized initial conditions in the sense that at $t=0$ the absolute value of the riemannian curvature at each point is at most one, and the volume of any metric ball of radius one is at least half that of the unit ball in $\mathbb{R}^{3}$. This is achieved by rescaling the metric and time by inverse positive constants as in Equation 2, From now on we implicitly assume that all flows under consideration have normalized initial conditions.

Definition 4.2.1. An $\epsilon$-neck in a riemannian 3-manifold $(X, g)$ centered at $x \in X$ is a region $T$ of $X$ with $x \in T$ and a diffeomorphism $\varphi: T \rightarrow S^{2} \times\left[-\epsilon^{-1}, \epsilon^{-1}\right]$ carrying $x$ into $S^{2} \times\{0\}$ such that the pushforward under $\varphi$ of $R(x)$ times $g$ is within $\epsilon$ in the $C^{N}$-topology of the product of the round metric of gaussian curvature $1 / 2$ on $S^{2}$ with the usual metric on the interval. See Fig. 2.

Claim 4.2.2 (Perelman [15]). There is $r>0$ such that the following holds. Let $(M, g(t))$ be a Ricci flow with normalized initial conditions defined for $0 \leq t<T$ with $M$ a closed, orientable 3-manifold. Then, for any point $(x, t)$ in the flow with $R(x, t) \geq r^{-2}$ one of the following holds: 


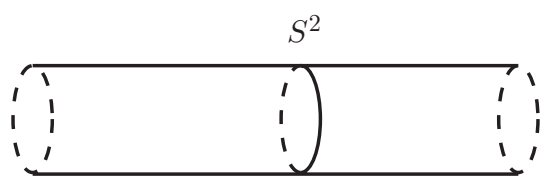

product

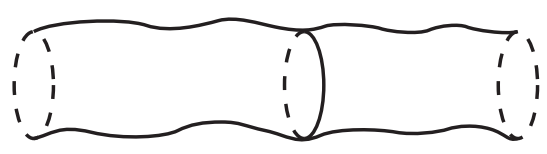

$\epsilon$-neck

FIGURE 2.

(1) the component of $M_{t}$ containing $(x, t)$ is diffeomorphic to a spherical spaceform.

(2) $(x, t)$ is the center of an $\epsilon$-neck in $M_{t}$.

(3) $(x, t)$ is contained in a submanifold of $M_{t}$ diffeomorphic to $D^{3}$ with every point in the boundary being the center of an $\epsilon$-neck in $M_{t}$. (Such a region is called an $\epsilon$-cap; see FIG. 3.)

4.3. Regions of High Curvature at the Singular Time. Now suppose that as $t \mapsto T$ the Ricci flow becomes singular. There is an extension of the above theorem to this time as well. There is an open subset $\Omega_{T} \subset M$ consisting of all the points where the riemannian curvature tensor remains bounded. By results of Hamilton 5] and Shi [21], there is a limiting metric on this open subset as $t \mapsto T$. We denote it $\left(\Omega_{T}, g(T)\right)$. Of course, the following is an immediate consequence of Claim 4.2.2

Claim 4.3.1. Then there are $r>0$ such that the following holds. Let $(M, g(t))$ be a Ricci flow with normalized initial conditions defined for $0 \leq t<T$ and becoming singular at $t=T$, with $M$ a closed, orientable 3-manifold. Then, for any point $(x, T) \in \Omega_{T}$ with $R(x, T) \geq r^{-2}$ one of the following holds:

(1) the component of $\Omega_{T}$ containing $(x, T)$ is diffeomorphic to a spherical spaceform.

(2) $(x, T)$ is the center of an $\epsilon$-neck in $\Omega_{T}$.

(3) $(x, T)$ is contained in an $\epsilon$-cap in $\Omega_{T}$.

In fact we can give more precise information about the ends of $\Omega_{T}$.

Definition 4.3.2. We fix $r>0$ and a surgery parameter $\delta>0$. Set $\rho=r \cdot \delta$. Let $\Omega_{T}(\rho)$ be the subset of $\Omega_{T}$ of points $(x, T)$ for which $R(x, T) \leq \rho^{-2}$ (see FIG. 4).

(1) An $\epsilon$-tube in $\Omega_{T}$ is a submanifold diffeomorphic to $S^{2} \times I$ such that each point is the center of an $\epsilon$-neck in $\Omega_{T}$.

(2) An $\epsilon$-circuit is a component of $\Omega_{T}$ that is a closed manifold every point of which is the center of an $\epsilon$-neck. Such a manifold is automatically diffeomorphic to $S^{2} \times S^{1}$.

(3) An $\epsilon$-horn (with respect to $r$ and $\delta$ ) is a closed subset $H \subset \Omega_{T}$ diffeomorphic to $S^{2} \times[0,1)$ with boundary contained in $\Omega_{T}(\rho)$ such that every point of

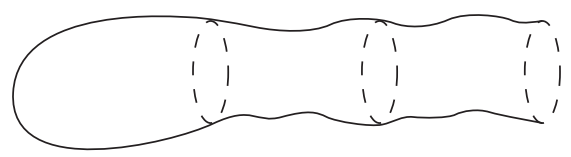

Figure 3. $\epsilon$-cap. 


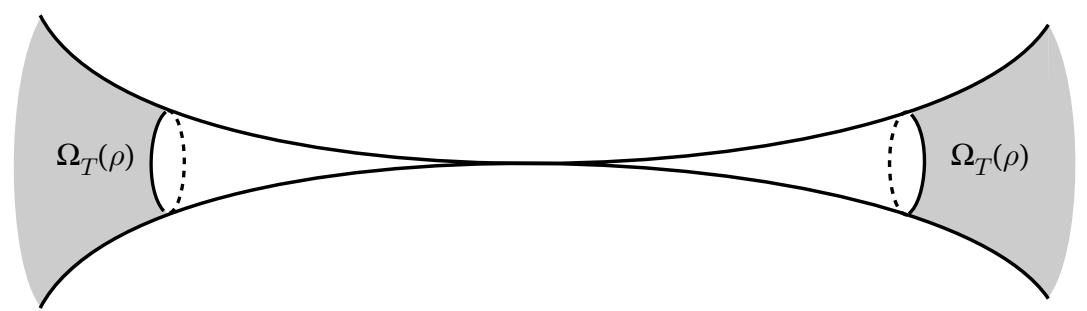

FiguRE 4.

$H$ is the center of an $\epsilon$-neck in $\Omega_{T}$. Of course, the scalar curvature goes to infinity at the other end of $H$.

(4) A double $\epsilon$-horn is a component of $\Omega_{T}$ diffeomorphic to $S^{2} \times(0,1)$ such that every point is the center of an $\epsilon$-neck in $\Omega_{T}$. The scalar curvature goes to infinity at each end of this component.

(5) A capped $\epsilon$-horn is a component of $\Omega_{T}$ diffeomorphic to $\operatorname{int}\left(D^{3}\right)$ such that each point is either the center of an $\epsilon$-neck or is contained in an $\epsilon$-cap. The scalar curvature goes to infinity near the end of a capped $\epsilon$-horn.

Claim 4.3.3 (Perelman [16]). There are $r>0$ and $\delta>0$ and $\rho=r \cdot \delta$ such that for any Ricci flow $(M, g(t))$ with normalized initial conditions ( $M$ a closed, orientable 3-manifold) becoming singular at $t=T$ the following holds. Any point $(x, T)$ in $\Omega_{T}$ with $R(x, T) \geq r^{-2}$ is contained in one of the following:

(1) a component of $\Omega_{T}$ that is diffeomorphic to a spherical space-form.

(2) an $\epsilon$-circuit diffeomorphic to $S^{2} \times S^{1}$.

(3) An $\epsilon$-tube with boundary components in $\Omega_{T}(\rho)$.

(4) An $\epsilon$-cap with boundary in $\Omega_{T}(\rho)$.

(5) An $\epsilon$-horn with boundary in $\Omega_{T}(\rho)$

(6) A capped $\epsilon$-horn.

(7) A double $\epsilon$-horn.

To treat singularities developing inside of tubes and caps, Perelman introduces a surgery along a 2-sphere. (Hamilton had earlier introduced in [8] a similar idea of surgery along 3-spheres in studying certain 4-dimensional Ricci flows.) This requires the introduction of what Perelman calls the standard solution.

4.3.1. The Standard Solution. The standard solution is a Ricci flow with initial conditions $\left(\mathbb{R}^{3}, g_{0}\right)$, where the metric $g_{0}$ is a $C^{\infty}$-metric that is the union of a metric on the upper hemisphere of $S^{3}$ close to the metric of constant positive curvature $1 / 2$ union the product metric on $S^{2} \times[0, \infty)$, with the metric on $S^{2}$ being the round metric of curvature $1 / 2$. We choose the metric so that the obvious $S O(3)$ symmetry is an isometric action. The unique fixed point $(p, 0)$ of this action is called the center of the standard solution. It turns out that, even though the initial riemannian manifold is not compact, the Ricci flow $\left(\mathbb{R}^{3}, g(t)\right)$ with these initial conditions is unique and is defined for all $t \in[0,1)$. Properties that this flow has that are important for our discussion are the following: For each $t \in(0,1)$, the metric $g(t)$ is complete and of bounded positive sectional curvature, asymptotic at infinity to the product of the metric of curvature $1 / 2(1-t)$ on $S^{2}$ with the standard metric on the real line, and the $S O(3)$-action is isometric for each $g(t), t<1$. See FIG. 5. 


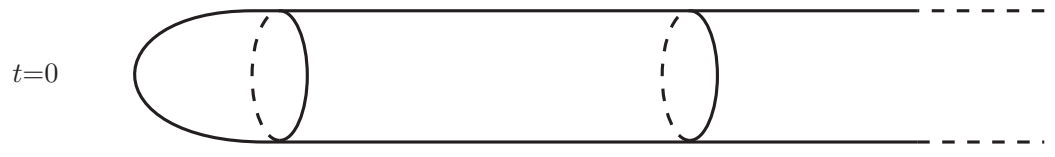

Figure 5. Standard solution.

4.3.2. Surgery. The idea now is to begin with the Ricci flow with normalized initial conditions and proceed to the singular time $T$. We remove all the components of $\Omega_{T}$ that are components of $M$ of the first two types in Claim 4.3.3. Also, we remove all components of $\Omega_{T}$ that are capped $\epsilon$-horns and double $\epsilon$-horns. Now in each $\epsilon$-horn it is possible to find a point $(x, T)$ that is the center of a $\delta(T)$-neck of radius $h(T)$. We cut $\Omega_{T}$ open along the central two-sphere of this $\delta(T)$-neck and delete the piece containing the end of the $\epsilon$-horn. Then we glue to the newly created boundary $S^{2}$ the ball of radius $\lambda$ from the initial conditions of the standard solution, where $\lambda$ is some fixed universal constant. We scale the metric on this latter ball by $h(T)^{2}$ so that the metrics on the two-spheres that we are gluing together are close to each other. We create a smooth metric on the union by using a partition of unity. See FIG. 6.

Most of the manifold $\left(M_{T}, g(T)\right)$ is the result of the time $T$ Ricci flow. But we have gone in and removed a neighborhood of the singularities that develop at this singular time and sewed in fixed riemannian metrics on 3-disks that we create by hand. Having made the closed riemannian manifold $M_{T}$ in this way, we use it as the initial conditions of a Ricci flow at time $t=T$. In this way we continue the Ricci flow with surgery to the next singular time. At the next singular time $T^{\prime}$, we do exactly the same surgery process, and then re-start the Ricci flow. In

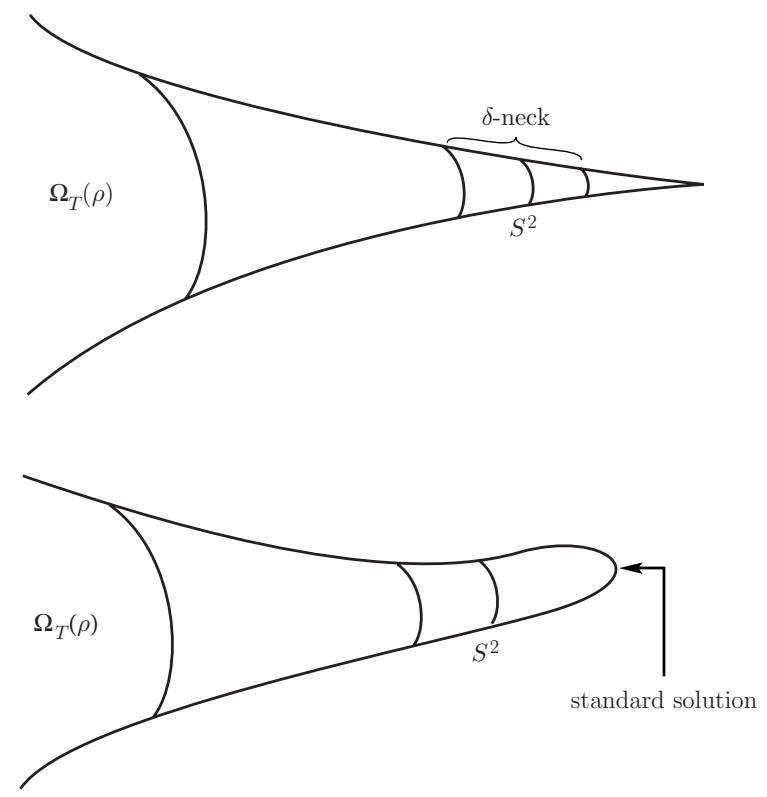

FIGURE 6. 

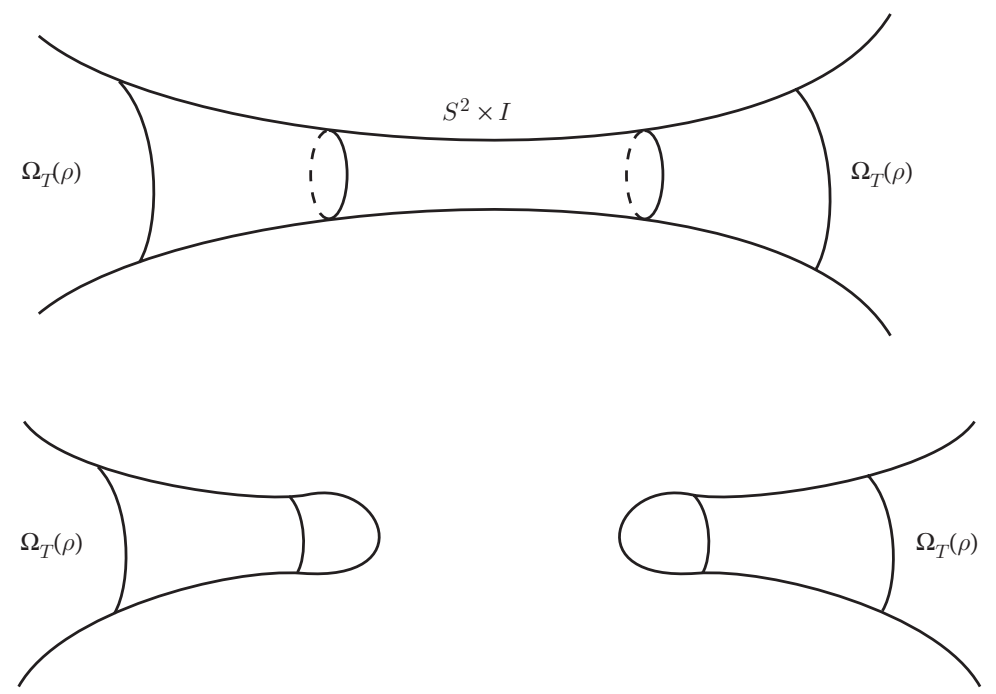

FiguRe 7. Tube surgery.

this way Perelman creates a Ricci flow with surgery with any compact riemannian 3 -manifold as initial conditions. This flow with surgery exists for all $t \in[0, \infty)$.

The 3-manifold changes topological type under the surgery process. The types of topological changes are threefold:

- (Tube Surgery) Remove a tube $S^{2} \times I$ from the manifold and glue in threedisks onto each end. (See Fig. 7.)

- (Cap Surgery) Remove a three-ball from the manifold and glue in another one. (See FIG. 8.)

- (Collapsing Component Surgery) A component of the manifold is entirely removed.
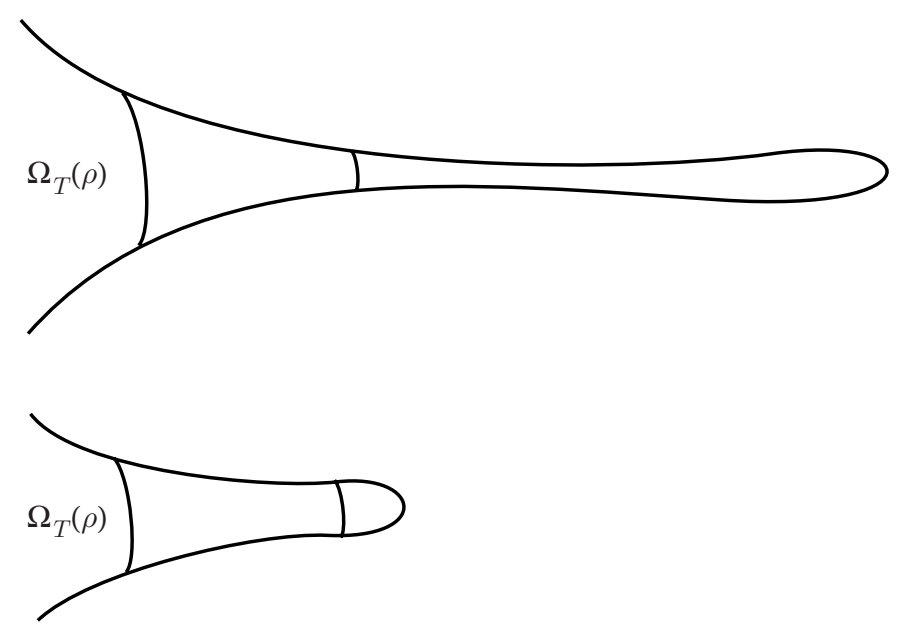

Figure 8. Cap surgery. 
The first type of operation is a usual topological surgery. This is how the connected sum decomposition required in the Geometrization Conjecture is implemented in the Ricci flow with surgery. (Of course, there is no reason to expect that every one of the surgeries produces a non-trivial connected sum decomposition. Some may simply split off $S^{3}$ 's, but this is harmless topologically.) The second type of operation is meaningful metrically since the balls in question have different metrics, but topologically it does nothing. The last type of operation may change the topology significantly since entire components are removed, but, as we have seen, each of these components is diffeomorphic to either a spherical space-form or to $S^{2} \times S^{1}$ and hence satisfies the conclusion of the Geometrization Conjecture. Thus, we are removing pieces of the prime decomposition of the original 3-manifold that satisfy the conjecture and keeping those about which we have not yet learned anything. From this description it is easy to see that if the result of the surgery process satisfies Thurston's Geometrization Conjecture, then so does the initial manifold.

Because the process of doing a surgery along an $S^{2}$ before any given time $T_{0}<\infty$ removes a fixed amount of volume from the 3-manifold (how much depends on the lower bound of the parameters $\delta(t)$ and $h(t)$ for $\left.t \leq T_{0}\right)$ and because under Ricci flow volume increases no faster than a fixed exponential rate of time, it follows that for any $T_{0}<\infty$ there can be only a bounded number of tube surgeries and cap surgeries before time $T_{0}$. This means that for all $t \leq T_{0}$ there is a uniform bound to the number of components that are created under these surgeries and hence a bounded number of components that can be removed by a collapsing component surgery. That is to say before time $T_{0}$ there are only finitely many surgeries. There may well be infinitely many surgeries as we allow time to go all the way to $\infty$. There will be only finitely many surgeries that remove non- $S^{3}$-components and only finitely many tube surgeries along essential $S^{2}$ 's. All the other surgeries will either be cap surgeries (which are topologically trivial), connected sum decompositions introducing new $S^{3}$ 's, or surgeries removing $S^{3}$-components. Let $(M, g)$ be a closed riemannian 3-manifold. Let $M_{t}$ be the result of Ricci flow with surgery with initial conditions $(M, g)$ at time $t$. If we can show that for some $t$, the manifold $M_{t}$ satisfies the conclusion of Thurston's Geometrization Conjecture, then it follows that $M$ also satisfies the conclusion of this conjecture.

4.3.3. A comment on the surgery parameters. From the above description of surgery it is not clear why we need to allow the surgery parameters $\delta(t)$ and $h(t)$ to decay as $t$ increases. Let us give a brief indication of the reason. The constants $r, \delta, h$ in Claims 4.2 .2 and 4.3 .3 depend on estimates on the ratios of the volume to the cube of the radius of all small balls in the manifold. The estimate is first established for the manifolds resulting from Ricci flows with normalized initial conditions. One has to prove analogous estimates for the Ricci flow with surgery with normalized initial conditions, and it turns out that these estimates are weaker and in fact depend on the surgery parameters used up to that time. (We cannot apply the result to the Ricci flow beginning at the surgery time $T$ since there is no reason to expect this manifold is normalized.) Consequently, the constant $r$ in these results must be allowed to depend on $t$ and to tend to zero as $t \mapsto \infty$. This forces us to allow the constant $\delta$ to depend on time and to go to zero as $t \mapsto \infty$. In fact, these parameters are defined by a complicated interlocking induction on larger and larger blocks of time. 
4.4. Limits at Infinity. The next step in Perelman's argument is to analyze the limits of the Ricci flow with surgery as $t \mapsto \infty$. Once again in this part of the argument he is following the path initiated by Hamilton in [9], though now the situation is more complicated for two reasons: (i) one is considering Ricci flow with surgeries instead of a Ricci flow, and (ii) there is no assumption of a bound on the (normalized) curvature. In spite of these complications Perelman claims that much of Hamilton's analysis can be adapted:

Claim 4.4.1 (Perelman [17]). Let $\left(M_{t}, g(t)\right)$ be a Ricci flow with surgery defined for $0 \leq t<\infty$.

- There is a finite collection of complete hyperbolic manifolds $H_{i}$ of finite volume and, for all sufficiently large $t$, an embedding

$$
\varphi_{t}: \coprod_{i} H_{i} \rightarrow M_{t}
$$

which, for sufficiently large $t$, is arbitrarily close to an isometry on an arbitrarily large compact subset of $\coprod_{i} H_{i}$ provided that we rescale the metric on $M_{t}$ by $c t^{-1}$ for an appropriate constant $c$ independent of $t$.

- For all $t>>1$ the tori of the $H_{i}$ parallel to infinity map to incompressible tori in $M_{t}$.

- For all $t>>1$, the complement of the image of a sufficiently large compact subset of $\bigsqcup_{i} H_{i}$ has a metric which is arbitrarily collapsed with lower curvature bounds.

For a point $x$ in a complete riemannian manifold define $r(x)$ to be the supremum of $r \geq 0$ such that the $R m(y) \geq-r^{2}$ on the metric ball $B_{r}(x)$ of radius $r$ centered at $x$. To say that a metric is $w$-collapsed at a point $x$ with lower curvature bounds means that $\operatorname{Vol}\left(B_{r(x)}(x)\right)<w r^{3}(x)$. A manifold is $w$-collapsed with lower curvature bounds if the above holds for every point $x$ of the manifold. To say the complement of the image of $\varphi_{t}$ is arbitrarily collapsed with lower curvature bounds as $t \mapsto \infty$ means that given any $w>0$ there is $T(w)<\infty$ such that $X_{t}=M_{t} \backslash \operatorname{Im}\left(\varphi_{t}\right)$ is $w$-collapsed with lower curvature bounds for all $t \geq T(w)$.

The fact that we have pieces of the $M_{t}$ isometrically approaching fixed hyperbolic pieces implies that all the surgeries done at sufficiently large times are done in the collapsed regions of the manifold $M_{t}$.

It follows from this claim that for any $t>>1$ we can separate $M_{t}$ along incompressible tori into pieces $\varphi_{t}\left(\amalg H_{i}\right)$ that are diffeomorphic to complete hyperbolic manifolds of finite volume and pieces $X_{t}$ where the metric is $w$-collapsed with lower curvature bounds where $w$ depends on $t$ and goes to zero as $t$ goes to infinity. It remains to show that for all $t>>1$ the prime factors of $X_{t}$ can be decomposed along incompressible tori and Klein bottles into pieces admitting finite volume locally homogeneous geometries.

4.5. The Collapsed Regions. To do this we invoke results from the theory of manifolds with collapsed metrics with lower curvature bounds. (These are generalizations of [2] where two-sided curvature bounds are assumed. For results on collapsing metrics with lower curvature bound, see for example [22] and [23].) In our situation, we have a family $X_{t}$ of 3-dimensional manifolds that are collapsing with (local) lower curvature bounds as $t \mapsto \infty$. The idea is to pass to a limit $X$ (of a subsequence of the $X_{t}$ ). This limit is a more general metric space than a 
riemannian manifold, but it is one in which the notion of curvature being bounded below still makes sense. The natural category for such objects is the category of Aleksandrov spaces; see [1] and [14]. Such spaces have a uniform dimension, and the dimension of the limit is most that of the manifolds in the sequence.

We apply this general theory to the limits arising from the collapsed regions of the Ricci flow with surgery as $t \mapsto \infty$. With one exception, what happens for these limits is that there are incompressible tori and Klein bottles that divide the collapsing regions into pieces. Each piece has an Aleksandrov space limit of either dimension one or two. Pieces where the Aleksandrov space limit is twodimensional are Seifert fibrations over hyperbolic orbifolds or are components that are nil-manifolds. Pieces where the Aleksandrov space limit is one-dimensional are components that are solv-manifolds or nil-manifolds or are $T^{2} \times I$ or the twisted $I$-bundle over the Klein bottle or $S^{2} \times I$. We do surgeries along the $S^{2} \times I$ pieces so as to remove them from the list. We can also remove from the list the $T^{2} \times I$ and twisted $I$-bundles over the Klein bottles by simply adding the cores of these pieces to the collection of tori and Klein bottles that we cut along. All the remaining pieces then are either solv-manifolds, nil-manifolds or Seifert fibered over hyperbolic twodimensional orbifolds. All of these manifolds have locally homogeneous metrics of finite volume. Thus, as before, we show that for all $t>>1$, every prime factor of $X_{t}$ can be decomposed along incompressible tori and Klein bottles in such a way that each piece has a locally homogeneous geometry of finite volume. The same statement then clearly holds for $M_{t}$ for all $t>>1$.

The one exceptional case that is not covered in the above description is the case of components $Y_{t}$ of the $M_{t}$ containing none of the hyperbolic pieces of $M_{t}$ and which, after rescaling so that its diameter is one, has volume bounded away from zero as $t \mapsto \infty$. With these rescalings and passing to a subsequence of such exceptional components $Y_{t}$ as $t \mapsto \infty$, we obtain a sequence of Ricci flows converging smoothly to a limiting Ricci flow. By results of Hamilton on curvature pinching [9], the sectional curvature of the limiting Ricci flow is everywhere non-negative. Furthermore, by the construction, the minimum of the sectional curvature is zero. Invoking the strong maximum principle of Hamilton for the Ricci flow [5], we conclude that the limiting Ricci flow is the constant flow on a flat manifold. Since the rescaled $Y_{t}$ converge smoothly to the limit, we conclude that for all $t>>1$ the $Y_{t}$ have flat metrics and hence satisfy Thurston's Geometrization Conjecture.

This then covers all cases and establishes that the conclusion of Thurston's Geometrization Conjecture holds for $M_{t}$ for all sufficiently large $t$. As we have already remarked, once we know the result for $t>>1$, we can go by downward induction past the surgery times to establish the result for the initial manifold of the Ricci flow with surgery. Since every closed, orientable topological 3-manifold has a normalized metric and hence can be used as the initial conditions for a Ricci flow with surgery with normalized initial conditions, it follows that Thurston's Geometrization Conjecture holds for all closed orientable 3-manifolds.

Strictly speaking, we have assumed that the orientable 3-manifold contains no embedded $\mathbb{R} P^{2}$. But as we have already remarked, any closed, oriented 3-manifold is a connected sum of a finite number of $\mathbb{R} P^{3}$ 's and a manifold with no embedded $\mathbb{R} P^{2}$. Establishing Thurston's Geometrization Conjecture for closed, orientable 3-manifolds with no embedded $\mathbb{R} P^{2}$ establishes it for all closed orientable 
3-manifolds. This then completes an outline of Perelman's approach to and claims about Thurston's Geometrization Conjecture.

4.6. Finite Time Extinction for Non-acyclic 3-Manifolds. There is still something interesting to say about the case of the Poincaré Conjecture and the Spherical Space-form question. We say that a Ricci flow with surgery becomes extinct at a finite time $T$ if $T$ is a surgery time and at $T$ all of the manifold is removed by the surgery process. According to Hamilton's theorem (3.3.1), this will be the case at the first surgery time if we begin with a manifold of positive Ricci curvature. In [17, Perelman claims that if $(M, g)$ are the initial conditions and if no prime factor of $M$ is acyclic (i.e., has a contractible universal covering), then the Ricci flow with surgery becomes extinct at some finite time. (There is a closely related result in [3] that can be used instead of [17].) In particular, if $\Sigma^{3}$ is a homotopy three-sphere, then all of its prime factors are also homotopy three-spheres and hence none of its prime factors is acyclic. Consequently, the Ricci flow with surgery becomes extinct in finite time. This means that in order to prove the Poincaré Conjecture or more generally to classify 3-manifolds with finite fundamental group and hence resolve the spherical space-form problem, one does not need the results on collapsing metrics from the previous subsection which are used to understand the topology as $t \mapsto \infty$ of the Ricci flow with surgery.

\section{Concluding Remarks}

I have stressed that aspect of Perelman's results that deal with Ricci flow with surgery. The reason of course is that these are the statements needed for topological applications. But, as Perelman himself makes clear, the most significant advances that he has made concern Ricci flow. I wish to summarize these. Perelman has managed a boot-strapping argument. First, he completely understands the gradient shrinking solutions in dimension three - there are only two types: a shrinking family of manifolds of constant positive curvature, and the cylinder $S^{2} \times \mathbb{R}$, where the family of metrics on the $S^{2}$ is a shrinking family of constant curvature metrics (or a manifold double covered by this one). Second, he is able to deduce compactness results for the space of non-flat, complete, ancient, volume non-collapsed solutions of non-negative curvature (modulo scaling). Third, he shows that every one of these solutions is either a family of shrinking constant positive curvature metrics or has in it larger and larger metric balls (as we go backwards in time) very close to the shrinking cylinder (or its quotient under a free involution). To establish these results, Perelman introduces the reduced length and reduced volume, and proves basic monotonicity results for them. Results on non-negatively curved ancient solutions are relevant to Ricci flow on compact 3-manifolds because of Hamilton's pinching result. Using this result, Hamilton showed that certain rescaling limits of regions of large curvature in a Ricci flow are non-flat, ancient solutions of bounded non-negative curvature. Perelman extended Hamilton's results in two ways. First, he showed that it was always possible to take such rescaling limits around all regions of large curvature and that such rescaling limits are automatically volume non-collapsed (this is another application of the reduced length function). Hence, all rescaling limits centered at points of high curvature fit into his classification scheme. This is the basis for Perelman's understanding of singularity formation (which occurs as the curvature goes to infinity) and the resulting surgery process. I have formulated things here for Ricci flow, and these results are used in order 
to define the surgery process. Nevertheless, as Perelman says, the extension of these results from Ricci flow to Ricci flow with surgery, while "technically quite complicated, involves no essentially new ideas."

Perelman's picture of the effect of Ricci flow with surgery fits perfectly with Thurston's Geometrization Conjecture. The surgeries at finite time implement the connected sum decomposition into prime factors and remove the prime factors that are non-acyclic, i.e., those whose universal covering is either $S^{3}$ or $S^{2} \times \mathbb{R}$. Then the decomposition along tori and Klein bottles occurs in analyzing the limits as $t \mapsto \infty$. The first decomposition is along tori and Klein bottles into inflating hyperbolic pieces and collapsing pieces. The metrics produced by the Ricci flow on the hyperbolic pieces, appropriately rescaled, converge to the hyperbolic metric. The pieces where the metrics are collapsing are studied through the theory of Aleksandrov spaces, i.e., the study of spaces with curvature bounded below. There is currently no understanding of the nature of the metrics produced by the Ricci flow on these pieces beyond the collapsing criterion. Rather, we obtain topological not metric conclusions from the Ricci flow and Aleksandrov space theory. Fortunately, these conclusions are strong enough to allow classification of the topological types, and all these topological types are known to possess locally homogeneous metrics (essentially by induction on dimension).

One open question, related to what is happening under the Ricci flow in the collapsed regions, is whether in general there are only finitely many surgeries for all positive time for Ricci flow with arbitrary initial conditions.

\section{REFERENCES}

[1] Y. Burago, M. Gromov and G. Perelman, "A. D. Aleksandrov spaces with curvature bounded below," Russian Math. Surveys 47 (1992), 1-58. MF 1185284 (93m:53035)

[2] J. Cheegar and M. Gromov, "Collapsing Riemannian manifolds while keeping their curvatures bounded, I, II." J. Differential Geom. 23 (1986), 309-346; 32 (1990), 269-298. MR.0852159 (87k:53087); MR1064875 (92a:53066)

[3] T. Colding and W. Minicozzi, "Estimates for the extinction time for the Ricci flow on certain 3-manifolds and a question of Perelman," arXiv.math.DG/0308090, August 25, 2003.

[4] D. Cooper, C. Hodgson, S. Kerchoff, 3-dimensional orbifolds and cone-manifolds, Math. Soc. Japan Memoirs, Vol. 5, Tokyo, 2000. MR1778789 (2002c:57027)

[5] R. Hamilton, "Three-manifolds with positive Ricci curvature," J. Differential Geom. 17 (1982), 255-306. MR0664497|(84a:53050)

[6] R. Hamilton, "The Harnack estimate for the Ricci flow," J. Differential. Geom. 37 (1993), 225-243. MR.1198607 (93k:58052)

[7] R. Hamilton, "Formation of singularities in the Ricci flow," Surveys in Differential Geometry 2, 7-136, International Press, 1995. MR.1375255 (97e:53075)

[8] R. Hamilton, "Four-manifolds with positive isotropic curvature," Comm. Anal. Geom. 5 (1997), 1-92. MR 1456308 (99e:53049)

[9] R. Hamilton, "Non-singular solutions of the Ricci flow on three-manifolds," Comm. Analysis and Geometry 7 (1999), 695-729. MR1714939 (2000g:53034)

[10] J. Hempel, 3-manifolds, Ann. of Math. Studies, Vol. 86, Princeton University Press, 1976. MR0415619(54:3702)

[11] H. Kneser, "Geschlossene Flächen in drei-dimesnionalen Mannigfaltigkeiten," Jahresber. Deutsch. Math. Verbein 38 (1929), 248-260.

[12] J. Milnor, "Groups which act on $S^{n}$ without fixed points," Amer. Journal of Math. 79 (1957), 623-630. MR0090056 (19:761d)

[13] J. Milnor, "Towards the Poincaré Conjecture and the classification of 3-manifolds," Notices AMS 50 (2003), 1226-1233. MR2009455 (2004h:57022) 
[14] G. Perelman, "Spaces with curvature bounded below," Proceedings of ICM 1994, 517-525, Birkhäuser, 1995. MR 1403952 (97g:53055)

[15] G. Perelman, "The entropy formula for the Ricci flow and its geometric applications," arXiv.math.DG/0211159, November 11, 2002.

[16] G. Perelman, "Ricci flow with surgery on three-manifolds," arXiv.math.DG/0303109, March 10, 2003.

[17] G. Perelman, "Finite extinction time for the solutions to the Ricci flow on certain threemanifolds," arXiv.math.DG/0307245, July 17, 2003.

[18] H. Poincaré, "Cinquième complément à l'analysis situs," Rend. Circ. Mat. Palermo 18 (1904), 45-110. (See Oeuvres, Tome VI, Paris, 1953, p. 498.) MR1401792 (98m:01041)

[19] P. Scott, "The geometries of 3-manifolds," Bull. London Math. Soc. 15 (1983), 401-487. MR.0705527|(84m:57009)

[20] H. Seifert, "Topologie dreidimensionaler gefaserter Räume," Acta Math. 60 (1933), 147288.

[21] W-X. Shi, "Deforming the metric on complete riemannian manifolds," J. Differential Geom. 30 (1989), 223-301. MR,1001277 (90i:58202)

[22] T. Shioya and T. Yamaguchi, "Collapsing three-manifolds under a lower curvature bound," J. Differential Geom. 56 (2000), 1-66. MR.1863020|(2002k:53074)

[23] T. Shioya and T. Yamaguchi, "Volume collapsed three-manifolds with a lower curvature bound," arXiv.math.DG/0304472, April 15, 2003.

[24] W. Thurston, Three-dimensional Geometry and Topology, Vol. 1 (Silvio Levy, ed.) Princeton Math. Ser., vol. 35, Princeton Univ. Press, 1997. MR.1435975 (97m:57016)

Department of Mathematics, Columbia University, New York, New York 10027

E-mail address: jm@math.columbia.edu 